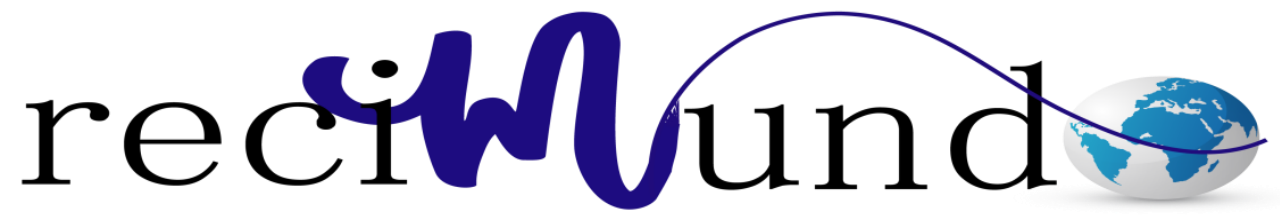

Revista Cientifica Mundo de la Investigación y el Conocimiento

Juan Francisco Chafla A. ${ }^{\text {a }}$ Santiago Silva ${ }^{\text {b; }}$ Alejandro Beltran ${ }^{\text {c; }}$ Hernán Barba ${ }^{\mathrm{d}}$

Tendencias tecnológicas para la modernización de centro de datos y propuesta de una arquitectura de data center moderno

Revista Científica Mundo de la Investigación y el Conocimiento. Vol. 2 núm., especial, mayo, ISSN: 2588-073X, 2018, pp. 3-30

DOI: 10.26820/recimundo/2.esp.2018.3-30

Editorial Saberes del Conocimiento

Recibido: 04/12/2017 Aceptado: 11/02/2018

a. Pontificia Universidad Católica del Ecuador, Quito Ecuador; jchafla390@ puce.edu.ec

b. Sinetcom S.A., Quito Ecuador; sdsilva@ outlook.com

c. Sinetcom S.A., Quito Ecuador; alejandrobp@ outlook.com

d. Politécnica Nacional del Ecuador; hernan.barba@ieee.org 


\section{Tendencias tecnológicas para la modernización de centro de datos y propuesta de una arquitectura de data center moderno}

Vol. 2, núm. 1., (2018)

Juan Francisco Chafla A; Santiago Silva; Alejandro Beltran; Hernán Barba

\section{RESUMEN}

En la actualidad, los negocios se están enfrentando a la era de la transformación digital; motivados por una nueva cultura de consumidores que usan la tecnología para aprovisionarse de servicios de manera rápida, ágil y transparente. El presente trabajo, entonces, se centra en entender la tecnología que apalanca la transformación digital que está marcando los negocios en la actualidad, y cuáles son las tendencias para la Modernización de los Centros de Datos que permiten a las empresas mantenerse competitivas dentro del mercado y estar preparadas para dicha transformación. Al final se presentará una propuesta de diseño de Arquitectura de Data Center Moderno basada en una plataforma Hyperconvergente, que recoja todas las características y recomendaciones de los primeros apartados, y que puede ser tomado como un modelo de referencia para aquellas empresas que se encuentra dando el paso hacia la modernización de su infraestructura tecnológica y así transformar su forma de hacer negocios.

Palabras claves: Infraestructura como Servicio (IaaS), Plataforma como servicio (PaaS), Solid State Drive (SSD), Software Defined Storage (SDS), Software Defined Datacenter (SDDC), Hyperconverged Infraestructure (HCI), Storage Area Network (SAN), Storage, Tansformación Digital, Transformación de TI, Modernización, Software Defined Network (SDN) y Software Defined Compute (SDC), Máquinas Virtuales (VMs). 


\title{
Tendencias tecnológicas para la modernización de centro de datos y propuesta de una arquitectura de data center moderno
}

Vol. 2, núm. 1., (2018)

Juan Francisco Chafla A; Santiago Silva; Alejandro Beltran; Hernán Barba

\begin{abstract}
Currently, businesses are facing the era of digital transformation; motivated by a new culture of consumers who use technology to procure services quickly, agilely and transparently. The present work, then, focuses on understanding the technology that leverages the digital transformation that is currently marking business, and what are the trends for the Modernization of Data Centers that allow companies to remain competitive within the market and Be prepared for this transformation. At the end, a design proposal for Modern Data Center Architecture based on a hyper-convergent platform will be presented, which will include all the characteristics and recommendations of the first sections, and which can be taken as a reference model for those companies that are giving the step towards the modernization of its technological infrastructure and thus transform its way of doing business.
\end{abstract}

Keywords: Infrastructure as a Service (IaaS), Platform as a Service (PaaS), Solid State Drive (SSD), Software Defined Storage (SDS), Software Defined Datacenter (SDDC), Hyperconverged Infrastructure (HCI), Storage Area Network (SAN), Storage, Digital Transformation, IT Transformation, Modernization, Software Defined Network (SDN) and Software Defined Compute (SDC), Virtual Machines (VMs).

\section{Introducción.}




\section{Tendencias tecnológicas para la modernización de centro de datos y propuesta de una arquitectura de data center moderno}

Vol. 2, núm. 1., (2018)

Juan Francisco Chafla A; Santiago Silva; Alejandro Beltran; Hernán Barba

De acuerdo a un estudio de IDC (International Data Corporation) titulado "Digital

Transformation Drives New IT Infraestructure Requirements", las tecnologías de tercera plataforma están estableciendo transformaciones fundamentales en los modelos de negocio que están en el core de las organizaciones. "Los líderes de los negocios están desafiados a mover sus empresas al siguiente nivel, una transformación digital del negocio, empleando tecnologías digitales acompañadas de innovación operacional y organizacional para crear nuevos modelos de negocio y manejar las oportunidades del mercado”. (IDC Custom Solutions, 2016)

Al conocer estos nuevos modelos de negocio y la importancia de la transformación digital de las empresas, muchas de ellas están ante la decisión de asumir dicha transformación, la cual se basa inicialmente en la creación de un plan de modernización de su Data Center considerando las tendencias tecnológicas actuales y de esa forma emprender un cambio estructural del área de TI para que ésta se convierta en el aliado estratégico de la empresa.

En el presente trabajo se exponen las principales tecnologías emergentes que apalancan la modernización de los Data Centers y consecuentemente preparan a las empresas para el proceso de transformación. De igual manera se presentará una propuesta de diseño de una arquitectura de Data Center Moderno basada en una plataforma HCI, la cual puede tomarse como modelo para aquellas empresas que están iniciando el proceso de transformación digital.

\section{Tecnologías Emergentes para Data Centers Modernos}




\section{Tendencias tecnológicas para la modernización de centro de datos y propuesta de una arquitectura de data center moderno}

Vol. 2, núm. 1., (2018)

Juan Francisco Chafla A; Santiago Silva; Alejandro Beltran; Hernán Barba

Las principales innovaciones tecnológicas que marcan a los Data Center modernos, y que surgieron como consecuencia de las limitaciones de los primeros, son las siguientes:

- Sistemas de Almacenamiento Monolíticos: los primeros centros de datos mostraban ineficiencias del uso de medios de almacenamiento para lo cual se desarrolló tecnología de almacenamiento monolítico en los cuales en lugar de provisionar espacio de almacenamiento directamente a cada servidor a través de sus discos locales, se crearon arreglos de discos externos que se presentaban a los servidores a través de la red LAN o SAN.

- Sistemas de Virtualización: La virtualización permite ejecutar múltiples cargas de trabajo simultáneamente en un hardware compartido y está basada en el "hypervisor" que es el software que abstrae los recursos físicos como CPU y memoria y los presenta como un pool de recursos disponibles a las máquinas virtuales

- Tecnología de discos Flash: Los sistemas de almacenamiento tipo flash, utilizan memorias no volátiles para almacenar la información cuyos tiempos de respuesta y de acceso a la información son varias veces más rápidos que su contraparte mecánica (discos de rotación).

- Tecnología de Convergencia e Hiperconvergencia: La convergencia es una arquitectura en donde toda la infraestructura de data center (comunicaciones, almacenamiento, cómputo, etc.) es concebida como un solo producto o equipo, permitiendo tener un punto centralizado de gestión de toda la plataforma, único punto de reporte de fallas, etc. Una solución de Hyperconvergencia es la evolución de las plataformas convergentes la cual provee servicios como virtualización de cómputo, redes, 
Tendencias tecnológicas para la modernización de centro de datos y propuesta de una arquitectura de data center moderno

Vol. 2, núm. 1., (2018)

Juan Francisco Chafla A; Santiago Silva; Alejandro Beltran; Hernán Barba

almacenamiento, servicios de datos, entre otros; apalancada en el modelo de Data Center

Definido por Software (SDDC)

- Tecnología de Cloud y SDDC: El Cloud o Cloud Computing, es un modelo que permite entregar infraestructura o aplicaciones de una manera flexible, rápida y bajo demanda. SDDC se apalanca enteramente en las infraestructuras virtualizadas a través de hypervisores como VMware, Hyper-V, etc. Capítulo 2: Arquitectura del Data Center Moderno

La arquitectura del Data Center moderno está construida en 3 capas lógicas y dos funciones que cubren estas capas, tal como se indica en la figura a continuación. Las tres capas son:

- Capa de Infraestructura Convergente e Hyperconvergente

- Capa de Infraestructura definida por Software

- Capa de Servicios 


\section{Tendencias tecnológicas para la modernización de centro de datos y propuesta de una arquitectura de data center moderno}

Vol. 2, núm. 1., (2018)

Juan Francisco Chafla A; Santiago Silva; Alejandro Beltran; Hernán Barba

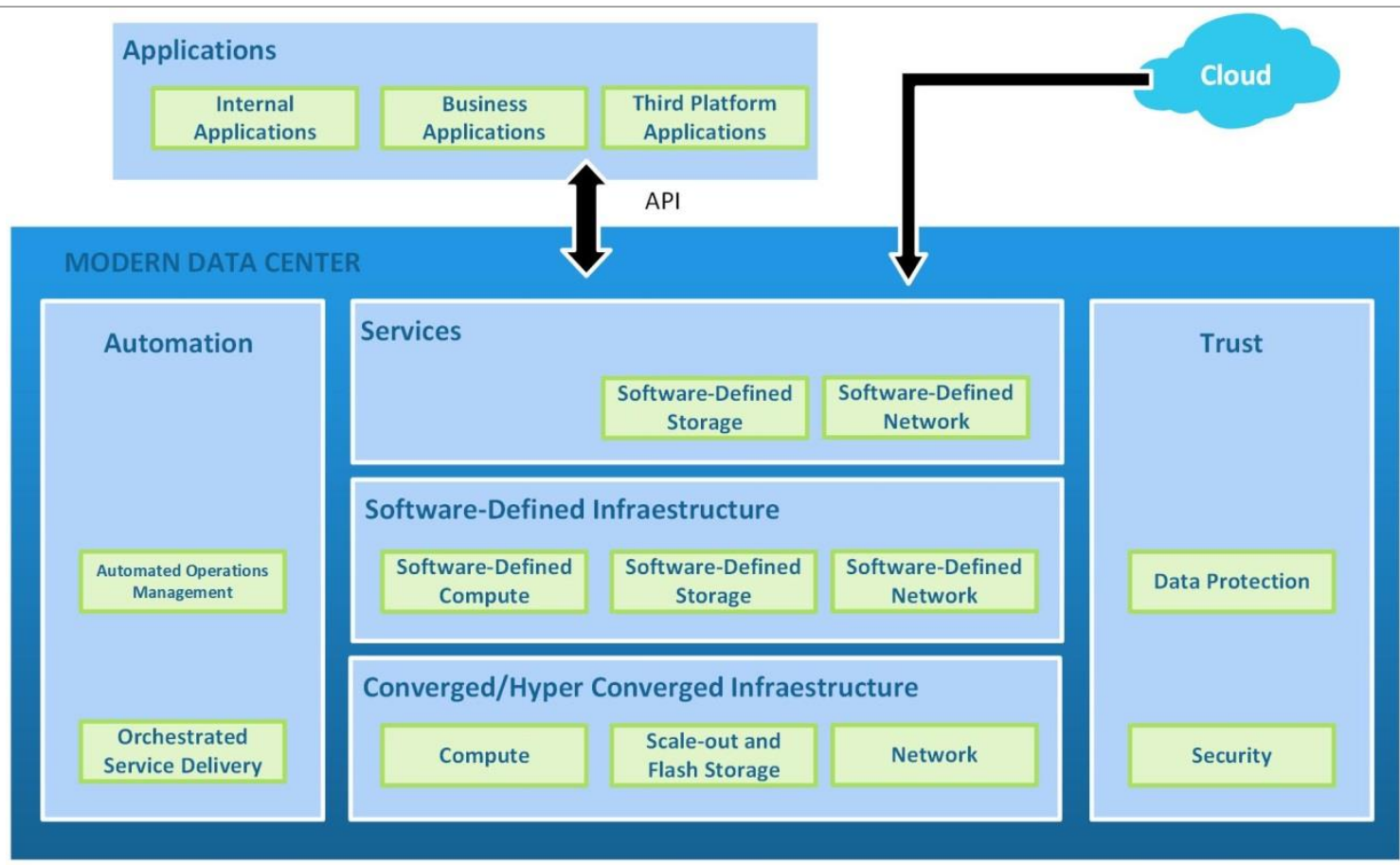

Figura 1. Arquitectura del Data Center Moderno (DELLEMC, 2017)

Cada una de estas capas tiene componentes de hardware y de software como se muestra en la figura. Las dos funciones mostradas también en la figura hacen referencia a la Automatización y a la Confianza. Estas dos funciones incluyen mecanismos y procesos que son necesarios para proveer agilidad al negocio, proteger la información y acceso seguro a las aplicaciones y servicios.

De igual manera el data center moderno permite la presentación de interfaces o APIs que habilitan a las aplicaciones a solicitar servicios de la infraestructura. Es crítico también que la infraestructura del data center moderno tenga capacidades de cloud, de tal manera que pueda desbordar cargas de trabajo de forma no disruptiva hacia las nubes públicas o privadas y de esta manera ahorrar costos a la empresa. 


\section{Tendencias tecnológicas para la modernización de centro de datos y propuesta de una arquitectura de data center moderno}

Vol. 2, núm. 1., (2018)

Juan Francisco Chafla A; Santiago Silva; Alejandro Beltran; Hernán Barba

A continuación se describirá cada uno de los bloques que constituyen la arquitectura del data center moderno que se mencionó previamente.

\section{Capa de Infraestructura Convergente e Hyperconvergente}

En el pasado, las organizaciones de TI construían su propia infraestructura tecnológica así como sus aplicaciones pero cada vez es menos común encontrar empresas que desean invertir ese modelo. Estas empresas desean una infraestructura fácil de desplegar sobre la cual puedan construir y ejecutar sus aplicaciones core para el negocio, de igual manera buscan plataformas sobre las cuales poder desplegar las aplicaciones de próxima generación. Las infraestructuras convergentes e hyperconvergentes cumplen con ambos requerimientos de las empresas modernas.

Una infraestructura convergente/hyperconvergente es un paquete pre-validado, preconstruido y pre-armado por el fabricante, que incluye cómputo, almacenamiento, redes y componentes de virtualización. Este tipo de soluciones ayuda a reducir el tiempo y el costo de implementación, configuración y gestión de la infraestructura de TI. Es esencial que las plataformas convergentes se construyan en base a almacenamientos all-flash y scale-out para que estén listas para soportar las aplicaciones de tercera plataforma.

\section{Capa de Infraestructura Definida por Software}

La capa de Infraestructura definida por software, en la práctica, es construida sobre la capa de infraestructura convergente/hyperconvergente. Esta capa permite instaurar un modelo basado en programación para controlar y gestionar los componentes y servicios de la 


\section{Tendencias tecnológicas para la modernización de centro de datos y propuesta de una arquitectura de data center moderno}

Vol. 2, núm. 1., (2018)

Juan Francisco Chafla A; Santiago Silva; Alejandro Beltran; Hernán Barba infraestructura convergente/hyperconvergente. Así mismo, permite separar las funciones de control de los componentes subyacentes de hardware y permite controlarlos desde un software externo.

Adicionalmente, el controlador basado en software abstrae todos los recursos físicos subyacentes de las aplicaciones; y permite el aprovisionamiento de componentes definidos por software tales como SDC, SDS, y SDN. Estos componentes definidos por software son accedidos por las aplicaciones como servicios.

Con una infraestructura definida por software las organizaciones pueden automatizar la configuración y el despliegue de servicios de TI, entregando una agilidad superior al negocio y un modelo más flexible basado en programación para gestionar los servicios.

\section{Capa de Servicios}

La capa de servicios está constituida por los medios necesarios para poder entregar recursos de TI a los usuarios finales para permitirles alcanzar los resultados esperados para el negocio sin que tengan que preocuparse por la infraestructura sobre la cual corre un servicio determinado.

. Esta capa incluye un "Catálogo de Servicios", el cual presenta la información acerca de todos los recursos de TI que son ofrecidos como servicios.

El portal de auto-aprovisionamiento, por su parte, provee el acceso al catálogo de servicios y facilita el auto-aprovisionamiento bajo demanda. La petición de auto- 


\section{Tendencias tecnológicas para la modernización de centro de datos y propuesta de una arquitectura de data center moderno}

Vol. 2, núm. 1., (2018)

Juan Francisco Chafla A; Santiago Silva; Alejandro Beltran; Hernán Barba

aprovisionamiento pasa a la capa de infraestructura definida por software la cual cumple con el requerimiento.

Ya que los usuarios pueden auto-aprovisionar recursos desde el portal de servicios de manera rápida, la capa de servicios mejora significativamente la agilidad del negocio.

\section{Automatización}

La automatización soporta las tres capas de la arquitectura de los data centers modernos. Esta capa contempla las siguientes funciones:

- Gestión automática de operaciones

- Entrega de servicios orquestados

Cuando un servicio es escogido del catálogo de servicios como lo muestra la figura a continuación, un flujo de trabajo asociado a éste es puesto en marcha. Basado en este workflow, el software de orquestación interactúa con los componentes de las capas de infraestructura definida por software y de infraestructura convergente/hyperconvergente para invocar las tareas de aprovisionamiento a ser ejecutadas por dichos componentes. 


\section{Tendencias tecnológicas para la modernización de centro de datos y propuesta}

de una arquitectura de data center moderno

Vol. 2, núm. 1., (2018)

Juan Francisco Chafla A; Santiago Silva; Alejandro Beltran; Hernán Barba

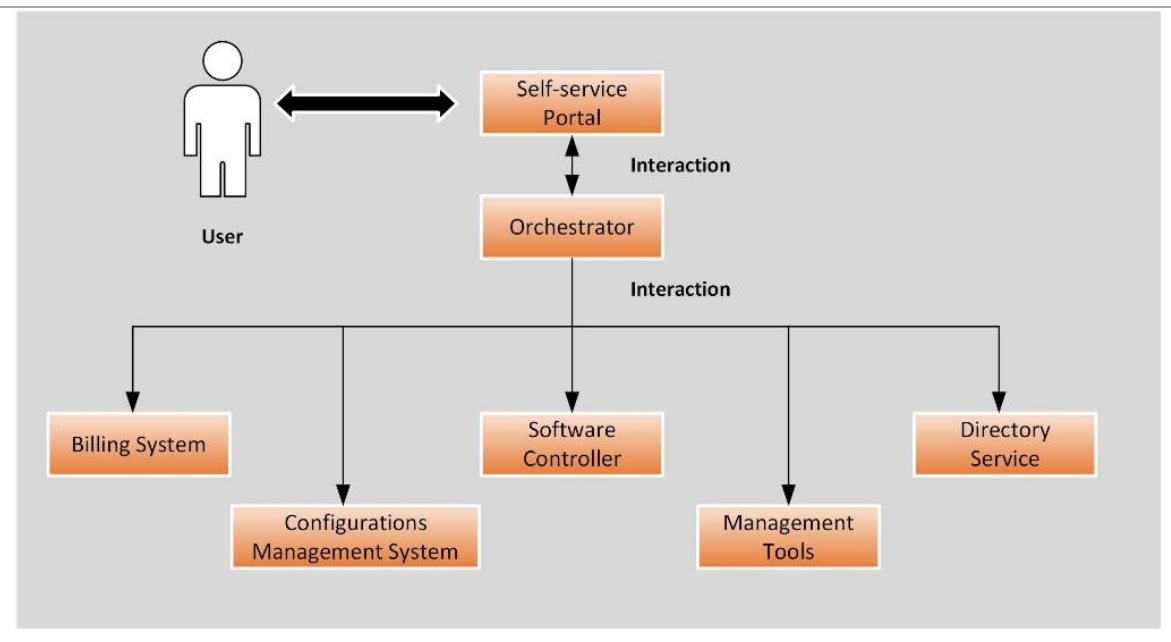

Figura 2. Arquitectura del Data Center Moderno (DELLEMC, 2017)

De esta forma, se aprovisionan los recursos apropiados de cómputo, red y almacenamiento, necesarios para cumplir con los requerimientos de los servicios. La automatización es una función necesaria que no solo reduce los costos administrativos sino que también provee la velocidad de ejecución requerida para agilizar el despliegue del servicio.

\section{Confianza}

Finalmente, las organizaciones deben ser capaces de confiar en la infraestructura de TI que poseen. La confianza depende del grado de control y visibilidad disponible al dueño de la información, por lo tanto asegurar los datos y proveerlos con alta disponibilidad son objetivos claves de la confianza.

La seguridad especifica la adopción de mecanismos técnicos y administrativos que mitiguen o minimicen las amenazas de seguridad y sean capaces de proveer un ambiente seguro al data center. Los mecanismos administrativos incluyen por ejemplo políticas de seguridad para 


\section{Tendencias tecnológicas para la modernización de centro de datos y propuesta de una arquitectura de data center moderno}

Vol. 2, núm. 1., (2018)

Juan Francisco Chafla A; Santiago Silva; Alejandro Beltran; Hernán Barba

el personal de TI o procedimientos estandarizados para dirigir la ejecución segura de varias operaciones. Por otro lado los mecanismos técnicos son usualmente implementados por herramientas o dispositivos desplegados a nivel de la infraestructura de TI.

La alta disponibilidad de información envuelve procedimientos, estrategias y disponibilidad de los recursos de TI apropiados para cada aplicación o servicio. Basado en la estrategia de alta disponibilidad de la información, se deberían implementar soluciones de protección de la información tales como respaldo y replicación de datos.

\section{Propuesta de Arquitectura de Data Center Moderno}

Las principales consideraciones tomadas para la propuesta de Arquitectura son:

- Crear una arquitectura orientada al SDDC, es decir que la mayor parte de los componentes considerados tengan la capacidad de adoptar un modelo definido por software

- Prescindir del uso de almacenamientos monolíticos y considerar una solución de almacenamiento definido por software o SDS

- Considerar la incorporación de soluciones tipo All-flash a nivel de almacenamiento

- Considerar arquitecturas de escalamiento tipo scale-out, es decir que ante un crecimiento de la infraestructura se mantenga la proporción de capacidad y performance constante.

- Considerar una arquitectura hiperconvergente por su simplicidad y agilidad es la recomendad en data centers modernos 


\section{Tendencias tecnológicas para la modernización de centro de datos y propuesta de una arquitectura de data center moderno}

Vol. 2, núm. 1., (2018)

Juan Francisco Chafla A; Santiago Silva; Alejandro Beltran; Hernán Barba

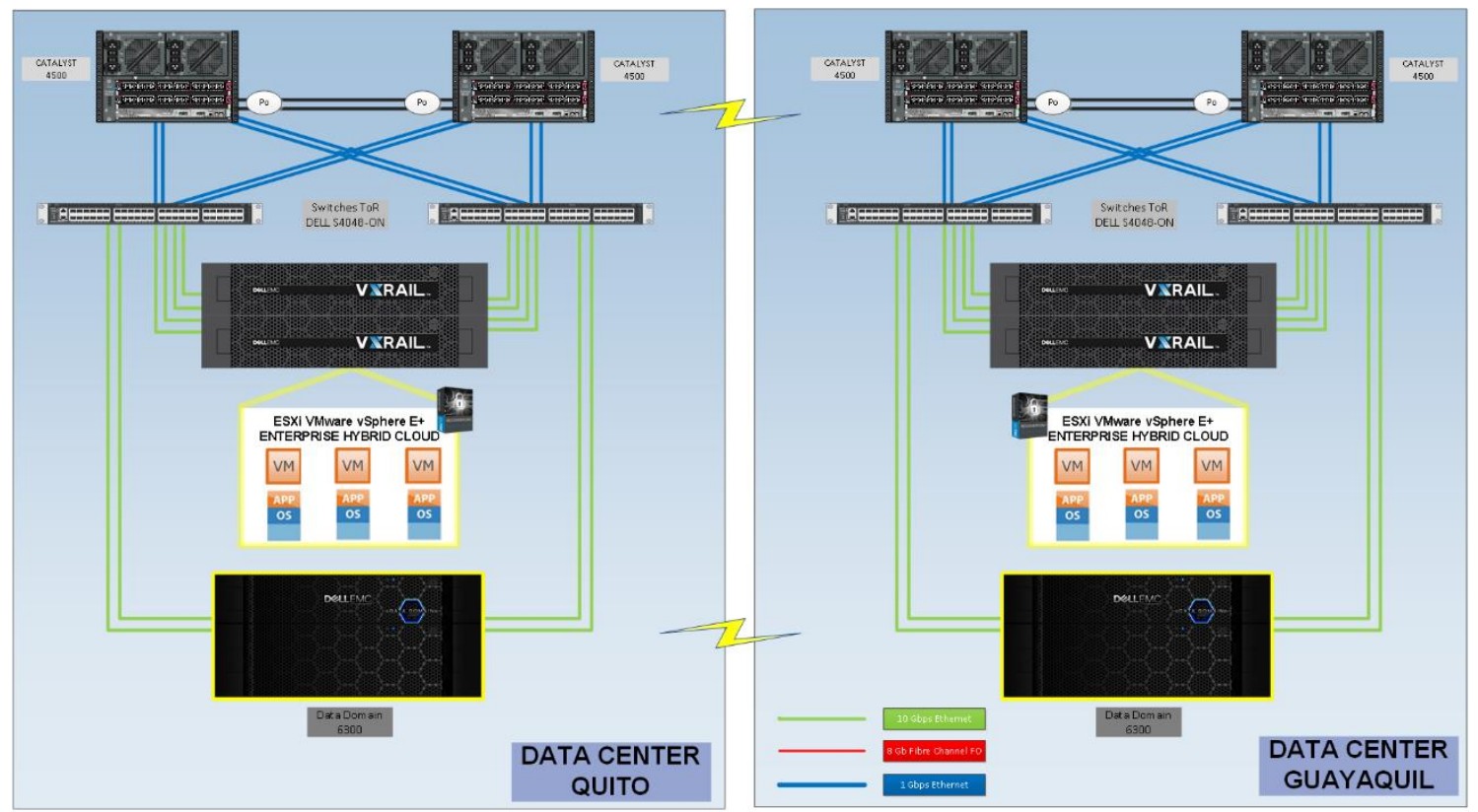

Figura 3. Diagrama de Arquitectura de Data Center Moderno

El diagrama de Arquitectura de Data Center moderno muestra la solución propuesta con cada uno de los componentes seleccionados. Como se puede observar, la arquitectura planteada contempla, no solo el sitio principal, que se asume estará en la ciudad de Quito, sino también el sitio contingente (Guayaquil) hacia donde será replicada la información de las VMs críticas para que el negocio siga operando en el caso de un desastre.

Básicamente la propuesta incluye los siguientes sistemas o componentes en el sitio principal y el contingente:

- Sistema Hiperconvergente: éste vendría a reemplazar los componentes de cómputo, almacenamiento, red SAN y virtualización de la infraestructura tradicional.

- Sistema de Switches Top of Rack: se trata de un par de switches de ultra baja latencia con conectividad a $10 \mathrm{Gbps}$ por fibra. 


\section{Tendencias tecnológicas para la modernización de centro de datos y propuesta de una arquitectura de data center moderno}

Vol. 2, núm. 1., (2018)

Juan Francisco Chafla A; Santiago Silva; Alejandro Beltran; Hernán Barba

- Sistema de Respaldo de la Información: se trata de una solución de respaldo a discos que aplica técnicas de de-duplicación para optimizar el espacio a disco.

- Sistema de Replicación de Información: este es un sistema basado en software que permite replicar, a nivel de granularidad de VM, aquella información crítica desde sitio principal al sitio de contingencia.

- Sistema de Automatización: este sistema incluye herramientas a nivel de software que permiten en primer lugar orquestar todos los recursos de la infraestructura y así poder proveer a los usuarios finales un portal web de auto-aprovisionamiento de infraestructura.

En el sitio contingente de Guayaquil se cuenta con exactamente las mismas herramientas, ya que por política y disposición de la mayoría de empresas, el sitio alterno deberá ser un espejo, a nivel de infraestructura del sitio principal.

El componente central de la arquitectura planteada es sin duda el sistema Hyperconvergente, plataforma que está siendo acogida masivamente a nivel mundial. Esta plataforma está concebida para ofrecer una administración centralizada y simplificada, en lugar de tener varios sistemas de gestión por cada componente, como se tiene en la infraestructura tradicional Basa su arquitectura en SDS por lo que, en la propuesta de data center moderno es posible prescindir por completo del almacenamiento monolítico y de la red SAN tan complejos de administrar y mantener operativos.

Por encima de la solución hyperconvergente se ejecutará un hypervisor, basado en la misma herramienta con la que ya cuentan varias empresas a nivel mundial y con la que están 


\section{Tendencias tecnológicas para la modernización de centro de datos y propuesta de una arquitectura de data center moderno}

Vol. 2, núm. 1., (2018)

Juan Francisco Chafla A; Santiago Silva; Alejandro Beltran; Hernán Barba

familiarizadas; esto es VMware. Este hypervisor gestionará los recursos de la solución hyperconvergente y permitirá el despliegue y operación de máquinas virtuales.

Complementando la solución hyperconvergente, se encuentra la herramienta de replicación, basada netamente en software, ésta se ejecuta como máquina virtual sobre el hypervisor anteriormente indicado, y es capaz de tomar cada máquina virtual del sistio principal y replicarla, a través del canal WAN, hacia el sistema HCI del sitio contingente. Es importante destacar que esta herramienta no replica simplemente bit a bit la información sino que antes de enviarla al canal WAN deduplica y comprime la data con el fin de reducir el ancho de banda necesario.

Para la protección de la información a nivel de respaldos, se incluye una solución especializada de respaldo a disco con deduplicación. Si bien es cierto existen alternativas en el mercado de soluciones de respaldo de información, como por ejemplo las librerías de cintas, repositorios NAS, etc.; lo que se recomienda, ahora que se habla del salto hacia la modernización y transformación de TI, es un sistema que optimice los respaldos y permita guardar más en menos espacio. Son precisamente los sistemas de respaldo a discos que ofrecen estas ventajas.

Se ha considerado también, dentro de la arquitectura, las herramientas de automatización de la plataforma moderna. Estas herramientas, que están definidas por software también, son básicamente dos: el orquestador y el auto-aprovisionamiento. Con estas herramientas lo que se pretende es darle agilidad al data center, requerimiento fundamental de las empresas modernas, para que por ejemplo los desarrolladores puedan auto-aprovisionarse de recursos de TI en un par de minutos, contrario a lo que sucede ahora que este proceso puede tardar días. 


\section{Tendencias tecnológicas para la modernización de centro de datos y propuesta de una arquitectura de data center moderno}

Vol. 2, núm. 1., (2018)

Juan Francisco Chafla A; Santiago Silva; Alejandro Beltran; Hernán Barba

Por último, y no menos importante, se tiene a los elementos de conectividad LAN; los cuales son un par de switches en alta disponibilidad con manejo de ultra baja latencia (a nivel de nanosegundos) y velocidades de línea, non-blocking, de $10 \mathrm{Gbps}$. Con esto se asegura una gran velocidad en el flujo de información entre el sistema hiperconvergente y los sistemas complementarios así como también alto rendimiento en el front-end (conexión hacia los usuarios finales).

\section{Diseño de la Solución HCI}

La solución central del Data Center moderno es la Plataforma HCI, por lo que en esta sección se muestra las etapas de diseño de esta infraestructura. El diseño y dimensionamiento de los otros componentes no se detalla. Para realizar el diseño se toman en cuenta los requerimientos típicos de una empresa mediana, sin embargo el proceso de diseño es personalizado por cada caso y empresa. El propósito de este trabajo es ser una guía o modelo más que un diseño ajustado a una empresa en particular. En resumen los parámetros útiles para dimensionar una solución HCI son los siguientes:

- Cantidad de Almacenamiento: 30TB usables en discos de estado sólido

- Performance: 10.000 IOPs

- Cantidad de máquinas virtuales: $80 \mathrm{VMs}$

- Cantidad de vCPU por VM: 4 vCPU por VM

- Cantidad de memoria RAM por VM: 10GB por VM 


\section{Tendencias tecnológicas para la modernización de centro de datos y propuesta de una arquitectura de data center moderno}

Vol. 2, núm. 1., (2018) Juan Francisco Chafla A; Santiago Silva; Alejandro Beltran; Hernán Barba

- Cantidad de almacenamiento por VM: 384GB por VM

La información de diseño mostrada arriba se ingresa en una herramienta, o sizer, como lo muestra la imagen a continuación.

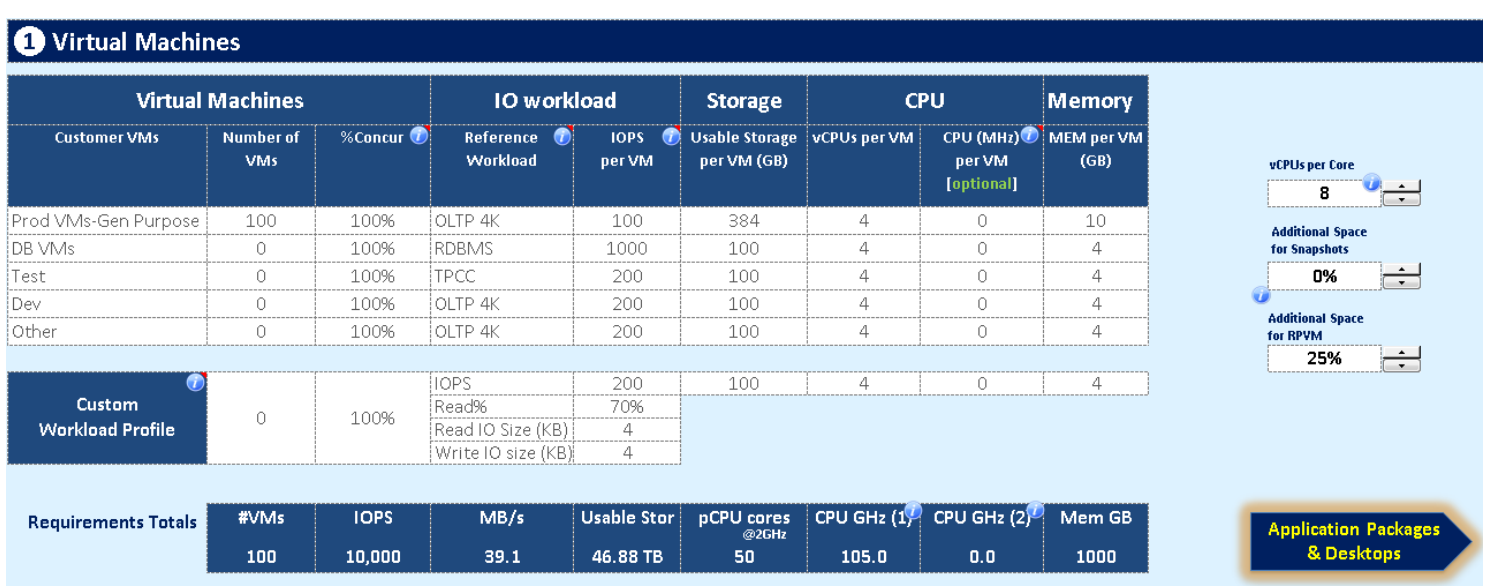

Figura 4. Sizer de la solución de HCI - Paso 1

Se eligen parámetros básicos de diseño como aplicación de técnicas de duplicación, factores de compresión, tipo de arreglo de discos, etc., tal como se muestra en la siguiente figura. 


\section{Tendencias tecnológicas para la modernización de centro de datos y propuesta de una arquitectura de data center moderno}

Vol. 2, núm. 1., (2018)

Juan Francisco Chafla A; Santiago Silva; Alejandro Beltran; Hernán Barba

\begin{tabular}{|c|c|c|}
\hline OPTIONS FOR 'ИSHAT-IF' COMPARISONS & OPTIONA & OPTION B \\
\hline Appliance Type (Hybrid/Hash) (1) & All Hash Only & Hybrid Only \\
\hline VxRail Model & G41F & G410 \\
\hline Rack Unit (1U1N, 2U1N, 2U4N) & Any Kit & Any Kit \\
\hline Max number of nodes in cluster (1) & 32 & 32 \\
\hline uSphere SW License & Enterprise Plus & Enterprise Plus \\
\hline Min CPU (GHz) per core & 2.0 & 2.0 \\
\hline Specific CPU Model & No & No \\
\hline Specific number of CPU sockets & No & No \\
\hline Support to GPU required (1) & No & No \\
\hline Min Memory per processor (GB) (C) & 64 & 64 \\
\hline Min Cache size (GB) & 400 & 400 \\
\hline Deduplication \& Compression & Yes & No \\
\hline Compression factor & Conservative & Conservative \\
\hline Min number of Disk Groups & 1 & 1 \\
\hline Min number Capacity Disks per DG & 1 & 1 \\
\hline Specific Capacity Disk size in TB & No & No \\
\hline Suggested Max I0 Utilization (\%) (1) & $70 \%$ & $70 \%$ \\
\hline Min \% spare storage capacity (C) & $25 \%$ & $25 \%$ \\
\hline Add resources for HA & No & No \\
\hline o of memory reserued (1) & $\mathbf{0} \%$ & $\mathbf{0} \%$ \\
\hline Choose a Fault Tolerance Method (1) & $\operatorname{RAIDS}(3+1)$ & Mirror FTI=1 \\
\hline Expected Cluster Growth (1) & $10 \%$ & $10 \%$ \\
\hline
\end{tabular}

Figura 5. Sizer de la solución de HCI-Paso 2

En la figura anterior se muestra el tercer paso de configuración. Aquí se puede elegir el tipo de CPU deseado por ejemplo un Intel Xeon E5-2660 v4, este campo se dejó en su valor por default para que la herramienta sugiera la mejor opción. La mayoría de parámetros se los dejo en sus valores por defecto a excepción de:

- VxRail Model: se configuró con el nodo G41F para forzar que la configuración sea con nodos de la Serie G modelo All Flash. La segunda opción (OPTION B) arrojará un diseño con nodos híbridos (G410) 


\section{Tendencias tecnológicas para la modernización de centro de datos y propuesta}

de una arquitectura de data center moderno

Vol. 2, núm. 1., (2018)

Juan Francisco Chafla A; Santiago Silva; Alejandro Beltran; Hernán Barba

- Compression Factor: como se mencionó en la explicación de vSAN, los nodos de Vxrail All-flash pueden realizar compresión de la Información. En este caso se configuró este parámetro como "Conservative" para no sobre valorar los niveles de compresión a los que se pueden llegar.

- Choose a Fault Tolerance Method: este parámetro se configuró en RAID5 (3+1). Es decir que la configuración mínima del clúster será de 4 nodos y que se puede perder todo un nodo sin afectar la operación del equipo ni la disponibilidad de la información.

- Expected CLuster Growth: aquí se definió un porcentaje de 10\% de crecimiento anual según es requerido por ASIACAR.

\begin{tabular}{|c|c|c|c|c|c|}
\hline \multirow[t]{3}{*}{ whorized portners. } & \multicolumn{5}{|c|}{5 nodes - VxRail G410F } \\
\hline & \multicolumn{5}{|c|}{$2 \times 2 \mathrm{U} 4 \mathrm{~N}$ appliances } \\
\hline & \multicolumn{5}{|c|}{ To red uce node count, increase ca U capacity per node } \\
\hline \multicolumn{6}{|l|}{ Node configuration } \\
\hline CPU & \multicolumn{5}{|c|}{$2 \times$ E- $2620 v 4-$ Total of $16 \times 2.1 \mathrm{GHz}$ cores $(33.6 \mathrm{GHz})$} \\
\hline Memory & \multicolumn{5}{|c|}{ 128GB per Proc / 256GB per node (8x32GB DIMMs) } \\
\hline Cache & \multicolumn{5}{|c|}{1 x DGs, each with 400 GB ME SSDs - (2.8\% ratio) } \\
\hline Storage & \multicolumn{5}{|c|}{ Total of 4 x 3.84TB SSD (13.96TB) - 1 free capacity slots } \\
\hline Cluster capacity & $\begin{array}{l}\text { Required } \\
\text { Capacity }\end{array}$ & $\begin{array}{l}\text { Min } \\
\text { \#nodes }\end{array}$ & $\begin{array}{l}\text { Required } \\
\text { Spare/HA }\end{array}$ & $\begin{array}{l}\text { Excess } \\
\text { Capacity }\end{array}$ & $\begin{array}{l}\text { Total } \\
\text { Configured }\end{array}$ \\
\hline CPU (Cores) & 67.8 & $\mathrm{n} / \mathrm{a}$ & $\mathrm{n} / \mathrm{a}$ & $\mathrm{n} / \mathrm{a}$ & 80.0 \\
\hline $\mathrm{CPU}(\mathrm{GHz})$ & 142.3 & 5.0 & 0.0 & 25.7 & 168.0 \\
\hline Memory (GB) & 1268.1 & 5.0 & 0.0 & 11.9 & 1280.0 \\
\hline Raw Storage (TB) & 52.0 & 5.0 & 17.3 & 0.5 & 69.8 \\
\hline \multirow[t]{2}{*}{ Usable Storage (TB) } & 50.6 & $\mathrm{n} / \mathrm{a}$ & $\mathrm{n} / \mathrm{a}$ & 0.5 & 51.1 \\
\hline & \multicolumn{5}{|c|}{$\begin{array}{l}\text { Minimum of } 4 \text { nodes to satisty fault toleranoe method and Ha optiors } \\
\text { Storage reserved for mermory sueps: } 20006 \text { B } \\
\text { Total of } 10: 106 \text { b ports required }\end{array}$} \\
\hline 10 Workload & \multicolumn{5}{|c|}{$\begin{array}{l}11 \mathrm{~K} \text { IOps } / 42 \mathrm{MBps} / 70 \% \text { Reads } \\
\text { Minimum of } 1.06 \text { nodes needed for } 10 \text { s }\end{array}$} \\
\hline
\end{tabular}

\begin{tabular}{|c|c|c|c|c|}
\hline \multicolumn{5}{|c|}{16 nodes - VxRail G410 } \\
\hline \multicolumn{5}{|c|}{$4 \times 2$ U $4 N$ appliances } \\
\hline \multicolumn{5}{|c|}{ To red uce node count, use Space efficiency options } \\
\hline \multicolumn{5}{|c|}{$2 \times$ E- $2620 \mathrm{y} 4$ - Total of $16 \times 2.1 \mathrm{GHz}$ cores $(33.6 \mathrm{GHz})$} \\
\hline \multicolumn{5}{|c|}{ 64GB per Proc /128GB per node (4x32GB DIMMs) } \\
\hline \multicolumn{5}{|c|}{1 x DGs, each with 400 GB ME SSDs - (4.3\% ratio) } \\
\hline \multicolumn{5}{|c|}{ Total of $5 \times 2$ TB $7.2 \mathrm{~K}(9.1 \mathrm{~TB})$ - 0 free capacity slots } \\
\hline $\begin{array}{l}\text { Required } \\
\text { Capacity }\end{array}$ & $\begin{array}{l}\text { Min } \\
\text { \#nodes }\end{array}$ & $\begin{array}{l}\text { Required } \\
\text { Spare/HA }\end{array}$ & $\begin{array}{l}\text { Excess } \\
\text { Capacity }\end{array}$ & $\begin{array}{l}\text { Total } \\
\text { Configured }\end{array}$ \\
\hline 67.8 & $\mathbf{n} / \mathbf{a}$ & $n / a$ & $\mathrm{n} / \mathrm{a}$ & 256.0 \\
\hline 142.3 & 5.0 & 0.0 & 395.3 & 537.6 \\
\hline 1268.1 & 10.0 & 0.0 & 779.9 & 2048.0 \\
\hline 106.1 & 15.5 & 35.4 & 4.2 & 145.6 \\
\hline 50.6 & $\mathrm{n} / \mathrm{a}$ & $\mathrm{n} / \mathrm{a}$ & 2.1 & 52.7 \\
\hline \multicolumn{4}{|c|}{$\begin{array}{r}\text { Price difference } \\
\text { Option } B / A\end{array}$} & $73.34 \%$ \\
\hline \multirow{2}{*}{\multicolumn{5}{|c|}{$\begin{array}{l}\text { Minimum of } 3 \text { nodes to satisfy fault toleranoe method and HA } \\
\text { storgge reserved for memory suaps: } 200068 \\
\text { Total of } 32: 106 \mathrm{~b} \text { ports required }\end{array}$}} \\
\hline & & & & Total of $32 \mathrm{~s} 106 \mathrm{~b}$ ports required \\
\hline \multicolumn{5}{|c|}{$11 \mathrm{~K}$ IOps/42 MBps/ $70 \%$ Reads } \\
\hline
\end{tabular}

Figura 6. Resultado del Sizer de la solución de HCI 


\section{Tendencias tecnológicas para la modernización de centro de datos y propuesta}

de una arquitectura de data center moderno

Vol. 2, núm. 1., (2018)

Juan Francisco Chafla A; Santiago Silva; Alejandro Beltran; Hernán Barba

En la figura anterior se muestra el resultado del cálculo de la herramienta de sizing. Como se puede apreciar se presenta dos opciones de soluciones hiperconvergentes con Vxrail. La primera opción es con nodos All-flash y la segunda con nodos híbridos. La primera diferencia que resulta evidente entre ambas opciones es la cantidad de nodos necesarios: en all-flash se requieren apenas 5 nodos mientras que en híbrido se requerirían 16. La elección siempre fue allflash sin embargo se presenta este resultado como evidencia de porque es mejor optar por nodos de estado sólido.

En resumen el dimensionamiento calculado es el siguiente:

- 5 nodos G410F cada uno con las siguientes características

○ 2 procesadores E5-2620 v4

○ 16 cores físicos

- 256GB de memoria RAM

○ 1 disco de 400GB SSD para caché

○ 4 discos de 3.84TB SSD para capacidad (1 slot libre para futuro crecimiento)

- Las capacidades totales del clúster Vxrail serían las siguientes

○ 80 CPUs físicos

- 1.280 GB de memoria RAM 
Tendencias tecnológicas para la modernización de centro de datos y propuesta de una arquitectura de data center moderno

Vol. 2, núm. 1., (2018)

Juan Francisco Chafla A; Santiago Silva; Alejandro Beltran; Hernán Barba

○ $\quad$ 69.8TB de almacenamiento RAW

- 51.1TB de almacenamiento usable

- Componentes de Software:

- 10 licencias de VMware vSPhere Enterprise Plus

- 1 Licenciamiento de vCenter Server

○ 1 Licenciamiento de Vxrail Manager

- 1 Licenciamiento de vRealize Log Insight

Como se puede observar en la figura las capacidades calculadas del clúster sobre pasan los requerimientos mínimos por lo tanto esto es un diseño válido y ajustado a las necesidades de la empresa.

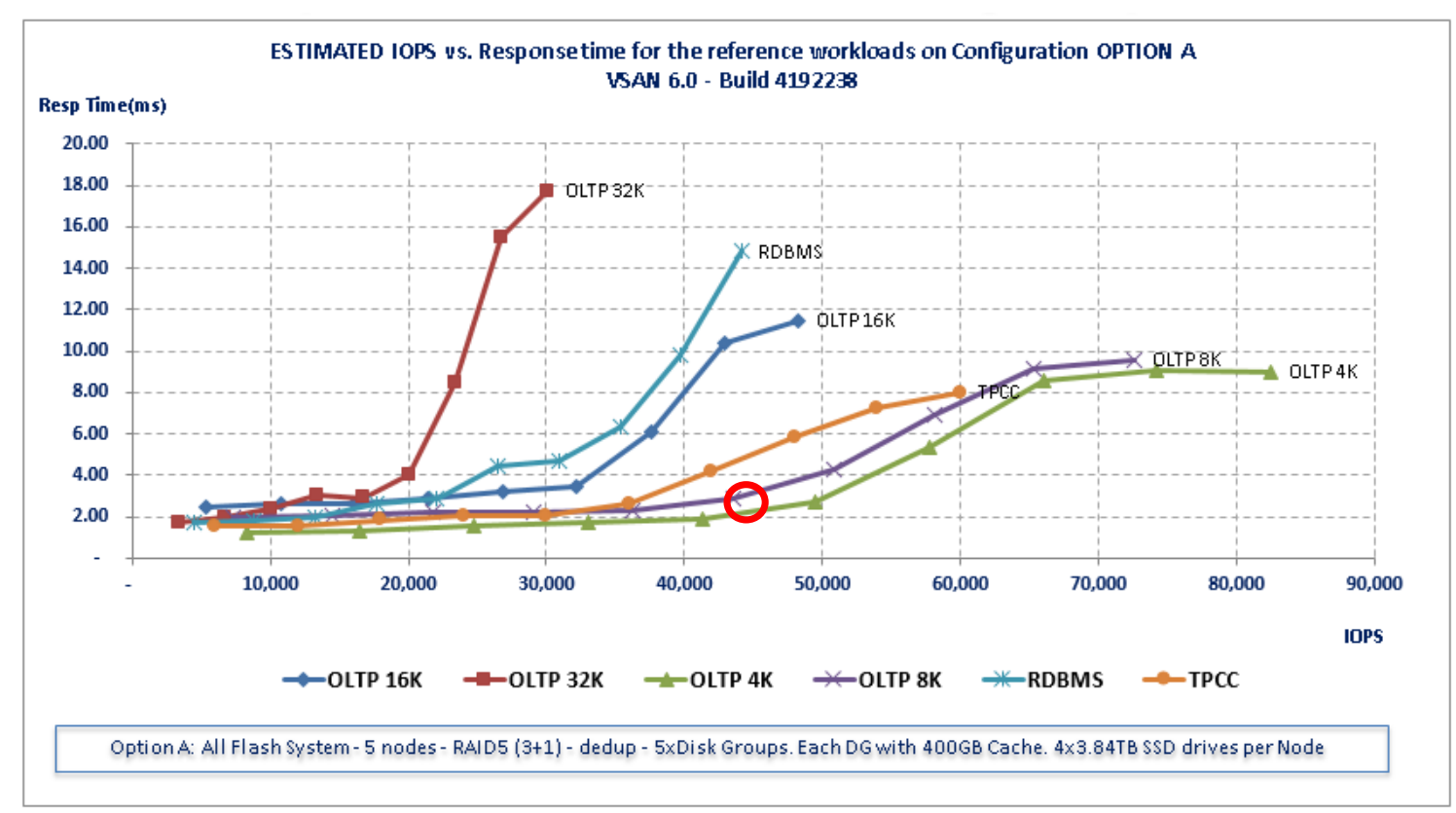




\section{Tendencias tecnológicas para la modernización de centro de datos y propuesta de una arquitectura de data center moderno}

Vol. 2, núm. 1., (2018)

Juan Francisco Chafla A; Santiago Silva; Alejandro Beltran; Hernán Barba

\section{Figura 7. Performance de la solución de HCI dimensionada}

En la figura anterior se puede observar un gráfico con las curvas de performance que tendría esta plataforma de $\mathrm{HCI}$ para distintos casos. Como la elección que se realizó es de bloques de almacenamiento de 4KB tipo OLTP se debe prestar atención a dicha curva (la verde). Esta curva nos muestra la variación del performance en IOPs de acuerdo al tiempo de respuesta del sistema. Considerando que los tiempos aceptables de respuesta de una aplicación crítica no deberían sobrepasar los 2 o 4 milisegundos, el performance que ofrece la solución diseñada es de 50.000 IOPs tal como se señala en la figura anterior.

Este performance es mucho mayor que el requerido de 10.000 IOPs, es precisamente una de las ventajas de trabajar con discos de estado de sólido que son capaces de multiplicar varias veces el desempeño de una solución tradicional.

\begin{tabular}{|c|c|c|}
\hline Componente & Cantidad & Descripción \\
\hline Sistema Vxrail & 1 & 1 Sistema HCI Vxrail All-Flash \\
\hline Appliances & 2 & $\begin{array}{l}2 \text { Appliances o chasís cada uno con: } \\
\text { - } 2 \text { UR de tamaño } \\
\text { - } 8 \text { nodos de capacidad } \\
\text { - } 5 \text { nodos G410 incluidos } \\
\text { - } 3 \text { bahías libres para nodos adicionales } \\
\text { - } 1.600 \text { Watts de potencia } \\
\text { - } 6.000 \mathrm{BTU} / \mathrm{h}\end{array}$ \\
\hline Nodos & 5 & $\begin{array}{l}5 \text { Nodos de la serie G410F All-flash cada uno con: } \\
\text { - } 2 \text { procesadores E5-2620 v4 } \\
\text { - } 16 \text { cores físicos } \\
\text { - } 256 \text { GB de memoria RAM } \\
\text { - } 1 \text { disco de 400GB SSD para caché } \\
\text { - } 4 \text { discos de } 3.84 \text { TB SSD para capacidad } \\
\text { - } 1 \text { slot libre para futuro crecimiento }\end{array}$ \\
\hline
\end{tabular}




\section{Tendencias tecnológicas para la modernización de centro de datos y propuesta de una arquitectura de data center moderno}

Vol. 2, núm. 1., (2018)

Juan Francisco Chafla A; Santiago Silva; Alejandro Beltran; Hernán Barba

\begin{tabular}{|c|c|c|}
\hline & & • 50.000 IOPs de desempeño \\
\hline Clúster & 1 & $\begin{array}{l}\text { 1 Clúster HCI con las siguientes capacidades: } \\
\text { - } 80 \text { CPUs físicos } \\
\text { - } 1.280 \text { GB de memoria RAM } \\
\text { - } 69.8 \mathrm{~TB} \text { de almacenamiento RAW } \\
\text { - } 51.1 \mathrm{~TB} \text { de almacenamiento usable }\end{array}$ \\
\hline Stack de Software & 1 & $\begin{array}{l}1 \text { Stack de software para SDDC con: } \\
\text { - } 10 \text { licencias de VMware vSPhere } \\
\text { Enterprise Plus } \\
\text { - } 1 \text { Licenciamiento de vCenter Server } \\
\text { - } 1 \text { Licenciamiento de Vxrail Manager } \\
\text { - } 1 \text { Licenciamiento de vRealize Log Insight } \\
\text { - } 15 \text { Licencias gratuitas de RecoverPoint }\end{array}$ \\
\hline
\end{tabular}

Tabla 1. Resumen de diseño de solución HCI propuesta

En la tabla anterior se puede ver un resumen del diseño y de los componentes que constituyen la solución de HCI diseñada.
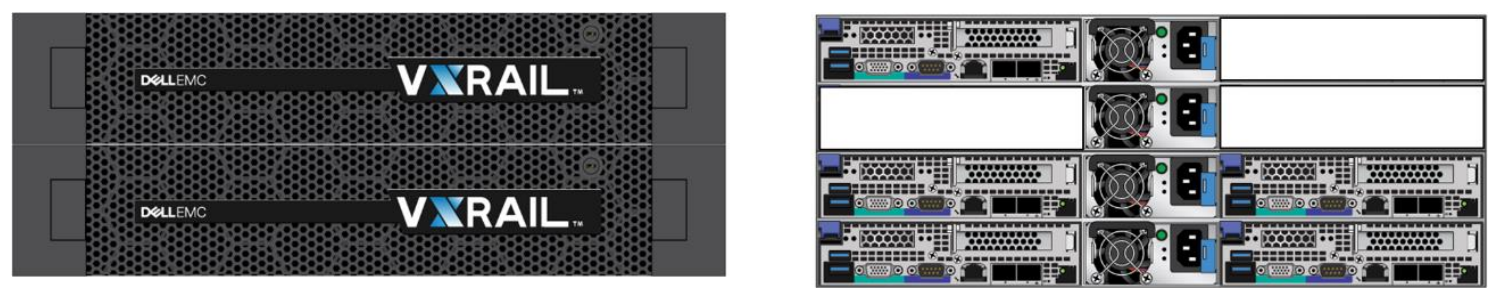

Figura 8. Vista Frontal y Posterior de la solución de HCI Diseñada

\section{Resumen de los componentes de la Arquitectura de Data Center Moderno}

En la siguiente tabla se puede apreciar el resumen de componentes considerados y recomendados para cumplir con cada uno de los pilares de los Data Center Modernos y que puede servir como modelo o base para aquellas empresas que están dando el salto hacia la Transformación Digital. 


\section{Tendencias tecnológicas para la modernización de centro de datos y propuesta de una arquitectura de data center moderno}

Vol. 2, núm. 1., (2018)

Juan Francisco Chafla A; Santiago Silva; Alejandro Beltran; Hernán Barba

\begin{tabular}{|c|c|}
\hline Componente & Descripción \\
\hline $\begin{array}{l}1 \text { VxRail Appliance con } 5 \text { nodos G410F all flash cada } \\
\text { uno con: } \\
\text { - } 2 \text { procesadores E5-2620 v4 } \\
\text { - } 16 \text { cores físicos } \\
\text { - } 256 \mathrm{~GB} \text { de memoria RAM } \\
\text { - } 1 \text { disco de 400GB SSD para caché } \\
\text { - } 4 \text { discos de 3.84TB SSD para capacidad }\end{array}$ & $\begin{array}{c}\text { Sistema Hyperconvergente } \\
\text { Propuesto }\end{array}$ \\
\hline 10 Licencias de VMware vSphere & $\begin{array}{l}\text { Licencias de Virtualizacion } \\
\text { para el sistema HCI }\end{array}$ \\
\hline $\begin{array}{l}2 \text { Switches ToR DELLEMC S4048-ON cada uno con: } \\
\text { - } 48 \text { puertos } 10 \mathrm{GbE} \mathrm{SFP+} \\
\text { - } 6 \text { puertos } 40 \mathrm{GbE} \\
\text { - } 2 \text { Fuentes de poder redundantes }\end{array}$ & $\begin{array}{c}\text { Switches ToR de conexión } \\
\text { Ethernet }\end{array}$ \\
\hline 100 licencias de RecoverPoint for Virtual Machines & Herramienta de Replicación \\
\hline $\begin{array}{l}\text { 1 Sistema Data Domain } 6300 \text { con: } \\
\text { - } 1 \text { Caja Principal con la controladora del equipo } \\
\text { y } 14 \text { TB } \\
\text { - } 1 \text { Caja de Expansió con 48TB configurados en } \\
\text { un RAID } 6 \text { de } 12+2 \text { discos de 4TB cada uno } \\
\text { - } 1 \text { Licencia de Replicación } \\
\text { - } 1 \text { Licencia de DDboost para deduplicación en } \\
\text { el origen } \\
\text { - } 5 \text { TB Licencia de Software de Respaldos } \\
\text { Networker }\end{array}$ & $\begin{array}{l}\text { Sistema de Respaldo de } \\
\text { Información a Disco con } \\
\text { Deduplicación }\end{array}$ \\
\hline $\begin{array}{l}\text { 1 Sistema de Automatización con: } \\
\text { - } 1 \text { Subscripción al stack de Software de EHC } \\
\text { - } 1 \text { Subscripción al software vCloud/vRealize } \\
\\
\text { - Suite } \\
\text { - } 1 \text { Subscripción al software VMware NSX }\end{array}$ & $\begin{array}{l}\text { Sistema de Automatización y } \\
\text { Autoaprovisionamiento }\end{array}$ \\
\hline
\end{tabular}

\section{Conclusiones.}

- La mayoría de empresas del entorno Ecuatoriano enfrentan grandes retos en la actualidad a nivel de plataforma tecnológica. Sus plataformas actuales tiene varios problemas entre ellos 


\section{Tendencias tecnológicas para la modernización de centro de datos y propuesta de una arquitectura de data center moderno}

Vol. 2, núm. 1., (2018)

Juan Francisco Chafla A; Santiago Silva; Alejandro Beltran; Hernán Barba

que muchos de sus componentes están cercanos al end-of-live y las cargas actuales de trabajo corren lentas sobre la infraestructura. Es una prioridad para estas empresas entrar en proceso de modernización tecnológica de su data center.

- Los data centers han evolucionado desde sus inicios y lo siguen haciendo. Es importante entender este proceso de evolución y la etapa actual para conocer las tendencias tecnológicas de las empresas modernas y exitosas. No se puede pretender modernizar un data center sin hacer un estudio de las principales tendencias y tecnologías que están marcando el camino de muchas empresas a nivel mundial.

- En el mundo de los negocios en el que se mueven las empresas actuales, está ocurriendo un proceso denominado transformación digital el cual está cambiando completamente la forma de hacer negocios al apalancar el negocio más en la tecnología para tener más llegada los usuarios finales. Todas las empresas que quiera sobrevivir en el ecosistema deberán pasar por este proceso, y el primer paso es la transformación de TI y modernización tecnológica de su Data Center.

- Una de las tendencias más marcadas actualmente en cuanto a tecnologías para el data center, es la adopción de Infraestructuras Hiperconvergentes (HCI), las cuales han demostrado ser muy versátiles, fáciles de operar, veloces para crecer y rápidas para reaccionar ante tan cambiantes requerimientos del negocio actual.

- Las infraestructuras HCI eliminan las islas que se creaban en los data centers tradicionales: isla de almacenamiento, isla de networking, isla de cómputo, etc., que tendían a hacer más 


\section{Tendencias tecnológicas para la modernización de centro de datos y propuesta de una arquitectura de data center moderno}

Vol. 2, núm. 1., (2018)

Juan Francisco Chafla A; Santiago Silva; Alejandro Beltran; Hernán Barba

complejo la administración y operación del data center. Con HCI todos los componentes anteriormente mencionados se condensan en unos equipos appliances con servidores commodity y el almacenamiento, cómputo y red es ahora manejado por software desde una misma consola centralizada e integrada con el hipervisor de virtualización.

- Otra de las tendencias tecnológicas que se tomó en cuenta para el diseño de la arquitectura de data center moderno propuesta, es la utilización de tecnologías All-flash o de 100\% drives de estado sólido. Se pudo estudiar que los discos mecánicos de rotación no han incrementado su velocidad desde el año 2000, es decir que han alcanzado su máximo desempeño físico. Por otro lado los drives SSD están tomando mucha fuerza a nivel mundial por su gran performance, ideal para las aplicaciones de tercera generación, y porque sus precios tienen una tendencia marcada a bajar hasta ser comparables con los costos de los discos mecánicos. Un SSD ofrece varias veces el performance de un disco mecánico.

- El modelo de Cloud público, privado o híbrido se está volviendo cada vez más atractivo para los negocios ya que permite incrementar la agilidad, bajar costos e incrementar la escalabilidad a niveles desconocidos.

- El SDDC (Software Defined Data Center) no es un dispositivo o tecnología específico, más bien es una forma de describir a los data centers modernos donde es posible abstraer la mayor parte de componentes físicos y poder controlarlos por software. El SDDC está caracterizado por la automatización, orquestación y la abstracción de recursos añadiendo una capa de software. 


\section{Tendencias tecnológicas para la modernización de centro de datos y propuesta de una arquitectura de data center moderno}

Vol. 2, núm. 1., (2018)

Juan Francisco Chafla A; Santiago Silva; Alejandro Beltran; Hernán Barba

- En las plataformas hyperconvergentes es posible alcanzar un nivel aceptable de desempeño gracias al uso de tecnologías de almacenamiento tipo flash. Los niveles superiores de IOPS que son capaces de entregar los discos flash, comparados con los discos mecánicos, permiten a las plataformas hyperconvergentes alcanzar los niveles de desempeño necesarios cuando los discos de rotación no son suficientes debido a sus limitaciones físicas de diseño.

- Es importante, para aquellas empresas, que estén empezando el proceso de transformación digital, el hacer un análisis de las tendencias tecnológicas a nivel mundial que están utilizando las empresas modernas y exitosas como por ejemplo Uber, Netflix, etc. Esto les dará una idea del tipo de plataforma que deben adquirir y el modelo tecnológico a desplegar. El presente documento pretende ser una guía para dichas empresas.

\section{Bibliografía.}

Amaris, Chris. Clayton, Marcus. Morimoto Rand. (Amazon Digital Services LLC). (2017). Building a Modern Datacenter in the Azure Cloud (Mini-Book Technology Series 4).

Geng, Hwaiyu. (John Wiley \& Sons). (2015). Data Center Handbook. Primera Edición.

Wu, Caesar. Buyya Rajkumar. (Morgan Kaufmann). (2015). Cloud Data Centers and Cost Modeling: A Complete Guide To Planning, Designing and Building a Cloud Data Center. Primera Edición

Arregoces, Mauricio. Portolani, Maurizio. (Cisco Press). (2003). Data Center Fundamentals.

Alger, Douglas. (Cisco Press). (2005). Build the Best Data Center Facility for Your Business. Primera Edición

Lowe, Scott. Green, James. Davis David. (Atlantis Computing). (2016). Building a Modern Data Center.

The Modern Data Center - Challenges and Innovations. (Mayo de 2015). es.commscope.com. Obtenido de http://es.commscope.com/Blog/The-Modern-Data-CenterChallenges-and-Innovations/ 
Tendencias tecnológicas para la modernización de centro de datos y propuesta de una arquitectura de data center moderno

Vol. 2, núm. 1., (2018)

Juan Francisco Chafla A; Santiago Silva; Alejandro Beltran; Hernán Barba

8 Steps to building a modern DataCenter. (Junio de 2013). www.wired.com. Obtenido de https://www.wired.com/insights/2013/06/8-steps-to-building-a-modern-datacenter/

The Pillars Of The Modern Data Center. (Marzo de 2016). blog.dellemc.com. Obtenido de https://blog.dellemc.com/en-us/pillars-modern-data-center/ 